\title{
Nanofluids and chemical highly retentive hydrogels for controlled and selective removal of overpaintings and undesired graffiti from street art
}

\author{
Rodorico Giorgi $^{1} \cdot$ Michele Baglioni $^{1} \cdot$ Piero Baglioni $^{1}$
}

Received: 31 January 2017 /Revised: 22 March 2017 / Accepted: 31 March 2017

(C) Springer-Verlag Berlin Heidelberg 2017

\begin{abstract}
One of the main problems connected to the conservation of street art is the selective removal of overlying undesired graffiti, i.e., drawings and tags. Unfortunately, selective and controlled removal of graffiti and overpaintings from street art is almost unachievable using traditional methodologies. Recently, the use of nanofluids confined in highly retentive pHEMA/PVP semi-interpenetrated polymer networks was proposed. Here, we report on the selective removal of acrylic overpaintings from a layer of acrylic paint on mortar mockups in laboratory tests. The results of the cleaning tests were characterized by visual and photographic observation, optical microscopy, and FT-IR microreflectance investigation. It was shown that this methodology represents a major advancement with respect to the use of nonconfined neat solvents.
\end{abstract}

Keywords Graffiti - Overpaintings · Selective removal · Street art $\cdot$ Chemical gels $\cdot$ Nanofluids

\section{Introduction}

Selective and controlled removal of graffiti and overpaintings from street art represents a completely novel issue in the conservation of cultural heritage, because it involves the removal of modern or contemporary paints from the same or similar materials, often applied without any concern about durability or on inappropriate substrates.

No kinship exists between Michele Baglioni and Piero Baglioni.

Rodorico Giorgi

giorgi@csgi.unifi.it

1 Department of Chemistry "Ugo Schiff" and CSGI, University of Florence, Via della Lastruccia 3, 50019 Sesto Fiorentino, Italy
In a broader sense, actually, this topic can include the removal of overpaintings in general, in modern or contemporary art, whenever the undesired material has a chemical nature similar to the one to be preserved. In this context, "selectivity" is the keyword, and the major aim of this process would be to limit the cleaning action to a few microns in depth, in order to remove the top undesired paint layer without altering or damaging the bottom original layer(s).

Chemical cleaning, often combined with some mechanical action (i.e., using abrasive systems) is the most traditional technique of graffiti removal [1-7]. In fact, several organic solvents, usually applied confined in poultices using absorbing materials, such as kaolin, sepiolite, or cellulose pulp, are able to solubilize or attack spray paint layers, which usually include synthetic polymers, such as alkyd, acrylic, or vinyl, as binding media [1]. However, in this context, traditional chemical methods do not provide enough control on the removal action.

Laser ablation can actually be selective for some specific applications, but, at present, the use of laser for the removal of graffiti or overpaintings [8-10] is still under investigation, since its application to this issue is not straightforward. Some encouraging results have most recently been obtained for the removal of overpaintings from oil easel paintings but, at the same time, Ciofini et al. state that with laser, there still exists "the need to control the ablation process because of the lack of intrinsic discrimination due to the high absorption of all organic materials in the UV region. Besides this limitation, the costs and maintenance issues of excimer lasers have impeded practical exploitation of their potential." [8].

Recently, the use of cleaning liquid systems confined in highly retentive chemical hydrogels was proposed [11-15]. In particular, nanofluids, such as micelles or microemulsions, proved to be very effective for this purpose. This methodology presents several advantages with respect to traditional approaches. First of all, toxicity and environmental impact of the cleaning 
systems are substantially reduced. To this aim, these systems are aqueous based and the amount of organic solvents is reduced to a few percens. Moreover, the confinement in highly retentive gels slows down the evaporation rate of volatile compounds. This is important especially in conservation of cultural heritage, where operators often work in inappropriate safety conditions, i.e., on great surfaces, in poorly ventilated environments, or without the due precautions (ventilated hoods, masks, etc.).

Secondly, nanofluid-loaded retentive chemical hydrogels enhance the control on the cleaning operation. The low amount of organic solvents, the reduced penetration rate in the porous substrate, and the fact that these gels are transparent (conservators can follow the cleaning process just by looking through them) guarantee that the action can be limited in space and time as wished.

Finally, chemical gels represent a considerable improvement with respect to traditional physical gels. In fact, physical gels are based on hydrophobic, electrostatic, van der Waals or hydrogen bond interactions, while the tridimensional polymer network of chemical gels is based on covalent bonds. Therefore, chemical gels have a coherent structure, and adhesion forces between the gel and the surface, which it is put in contact with, have a lower energy than cohesion forces inside the gel itself. This means that these gels can be simply and safely removed from the treated surface without rinsing or using extra mechanical action to clear gel residues. In fact, it has been shown that no significant residues due to the gel can be found on cleaned areas after the application [16].

Therefore, the only nonvolatile components of these systems, which can remain on the treated areas as residues, are surfactants, which can be easily cleared out using the same chemical hydrogels loaded with pure water, even if most of the formulations proposed include biodegradable nonionic surfactants, which are eco-friendly and prone to biodegradation over time, reducing the need for a thorough rinse of the treated area.

Here, we report on the first application of this methodology to the removal of overpaintings from mockup samples in laboratory tests. A previously formulated nanofluid, based on ethyl acetate and propylene carbonate [17-19], was combined with a poly(2-hydroxyethyl methacrylate (pHEMA)/ poly(vinylpyrrolidone) (PVP) semi-interpenetrated polymer network (sIPN) $[16,20,21]$ and used to selectively remove a white acrylic modern paint from a white acrylic paint laid on a mortar sample. Cleaning results were, then, characterized by means of visual and photographic observation, optical microscopy, and FT-IR microreflectance investigation.

\section{Materials and methods}

\section{Chemicals}

Sodium dodecylsulfate (SDS; Sigma-Aldrich, assay $\geq 99 \%$ ), 1-pentanol ( $\mathrm{PeOH}$; Merck, assay $\geq 98.5 \%$ ), ethyl acetate (EA;
Sigma-Aldrich, ACS reagents, assay $\geq 99.5 \%$ ), propylene carbonate (PC; Sigma-Aldrich, assay 99\%), 2-hydroxyethyl methacrylate (HEMA; Sigma-Aldrich, assay 97\%), PVP (Sigma-Aldrich, average $\mathrm{Mw} \approx 1300 \mathrm{kDa}$ ), $\alpha, \alpha^{\prime}$ azoisobutyronitrile (AIBN; Fluka, assay 98\%), and N,N-methylene-bisacrylamide (MBA; Fluka, assay 99\%) were used as received. Water was purified using a Millipore MilliRO-6 and MilliQ (Organex Systems) apparatus (resistance $>18 \mathrm{M} \Omega \mathrm{cm}$ ). Sand (50-70 mesh particle size) was purchased from SigmaAldrich, and aged slaked lime was purchased from La Banca della Calce s.r.l., Bologna, Italy.

\section{Modern paints}

White (Liquitex) and blue (Rembrandt, Royal Talens) tube paints were purchased and used as received for selective removal tests. Both paints are water-based emulsions. FT-IR analysis of these paints was performed in order to obtain information on their chemical composition (only a few data could be extracted from the technical sheets of the products), but transmission spectra are not shown for sake of conciseness (non-corrected microreflectance spectra of the two paints, however, are visible in Fig. 1). The main results of the analysis are reported hereafter. The white paint includes poly $(n$-butyl acrylate/methyl methacrylate) as the binder, while $\mathrm{TiO}_{2}$ is the pigment. $\mathrm{BaSO}_{4}$ is probably included in the paint formulation as an inert filler. On the other hand, the blue paint is characterized by poly(ethyl acrylate/methyl methacrylate) as the binder and artificial ultramarine blue as the pigment. Kaolin is also present as a filler. By FT-IR, it was not possible to identify surfactants, plasticizers, and other additives present in the two paints in smaller amounts.

\section{pHEMA/PVP semi-interpenetrated polymer network}

pHEMA/PVP semi-interpenetrated networks are obtained embedding chains of PVP in a network of pHEMA, which is formed through a polymerization reaction, as described by Domingues et al. [16]. The final properties of the hydrogel mainly depend on the pHEMA/PVP ratio and the amount of water used during the gelation process. By adjusting the formulation, it is possible to tune the properties of these gels (i.e., hydrophilicity of the network, pore size distribution, mechanical properties) according to specific application needs.

\section{Preparation of the nanofluid}

The nanofluid was prepared starting from a micellar solution of SDS and 1-pentanol in water. Then, propylene carbonate and ethyl acetate were slowly added under constant stirring, until an optically clear system was obtained. The composition of the final system (\% $w / w)$ is as follows: water, $73.3 \%$; SDS, $3.7 \%$; PeOH, 7\%; EA, 8\%; PC, $8 \%$. The nanofluid was then 


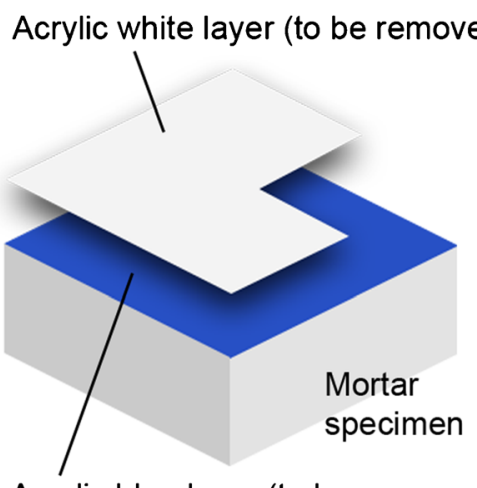

Acrylic blue layer (to be preserved)

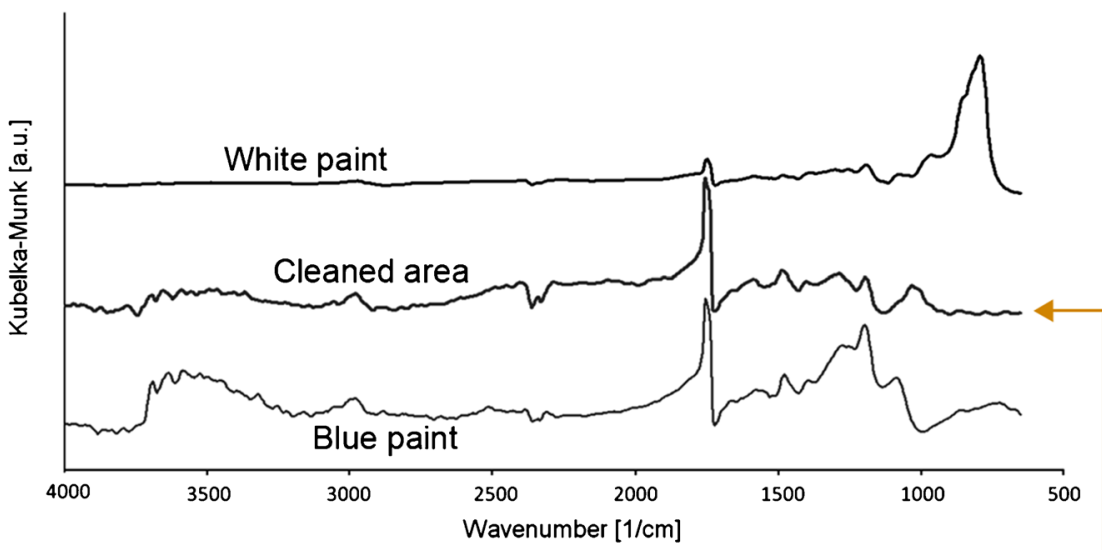

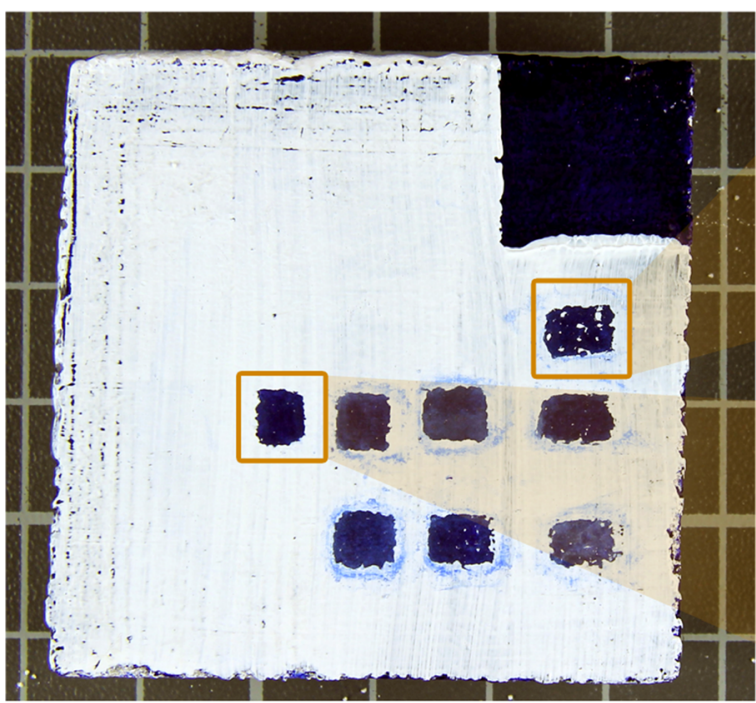

Fig. 1 Laboratory tests on selective removal of a layer of modern acrylic white paint from modern acrylic blue paint. Several spot tests were performed in order to find the optimum application time combination (first area tested from the left). On the same sample, another test area is

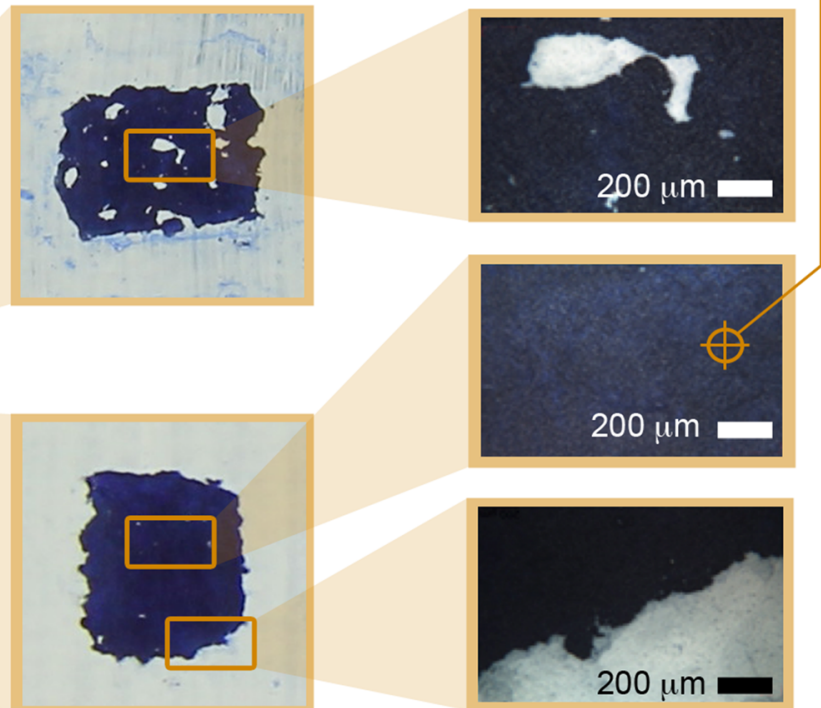

shown in the zoom box, where some white paint residues can be seen, indicating that application time in this case was not suited. Also, FT-IR microreflectance spectra are reported, which confirms that the cleaning in the investigated area was successful, as no white paint residues were found loaded into the gel simply by immersing the gel itself in the liquid and letting equilibrate overnight.

\section{Preparation of mockups}

Mortar mockups were prepared in laboratory mixing one part of slaked lime and three parts of sand. The mixture was then poured into wooden molds of $5 \mathrm{~cm} \times 5 \mathrm{~cm} \times 1.5 \mathrm{~cm}$ and let dry for 1 month, until setting of the mortar was enough to handle and treat the tiles. These were, then, painted with a layer of blue paint and let dry in oven at $60^{\circ} \mathrm{C}$ for 2 days, in order to speed up complete evaporation of water. Finally, the white paint was laid over the blue one, leaving a reference square uncovered. Removal tests were performed 2 weeks later, after complete setting.

\section{Selective removal tests}

Removal tests were performed with the nanofluid-loaded pHEMA/PVP gel. This was cut into small pieces (about $8 \times 8 \times 2 \mathrm{~mm}^{3}$ ), which were adhered on the surface of the white paint and let interact with the paint layer for a variable time ( $5 \mathrm{~s}$ to $1.5 \mathrm{~min}$ ). After gel removal, the swollen paint was gently removed, performing a soft mechanical action using a humid cotton swab.

\section{Optical microscopy observation}

A Reichert Zatopan 353-890 microscope was used to collect micrographs. The instrument was coupled with a Nikon Digital Sight DS-Fi2 camera. The NIS-ELEMENTS software was used to capture and edit images. 


\section{FT-IR microreflectance}

Microreflectance FT-IR analyses were performed using a Nexus Fourier transform infrared spectrometer from Nicolet. The instrument was interfaced with OMNIC software and equipped with a microscope for microanalysis. A MCT detector was used to collect the signal in the $4000-650-\mathrm{cm}^{-1}$ range. A gilded surface was used to collect the background signal. The spectra were collected as single-beam files as the sum of 128 scans with a resolution of $4 \mathrm{~cm}^{-1}$. Then, they were divided by the background signal and transformed using the Kubelka-Munk algorithm, which is commonly used to display reflectance spectra, as it applies a scaling factor to the curves in order to obtain data more easily comparable with the absorption spectra.

\section{Results and discussion}

Laboratory cleaning tests were performed in order to reproduce overpainted modern paint layers on mural paintings.

Some preliminary tests were carried out in order to evaluate the results of working with nonconfined neat organic solvents or even nanofluids. To this aim, some cleaning tests were performed using cotton swabs soaked with ethyl acetate,
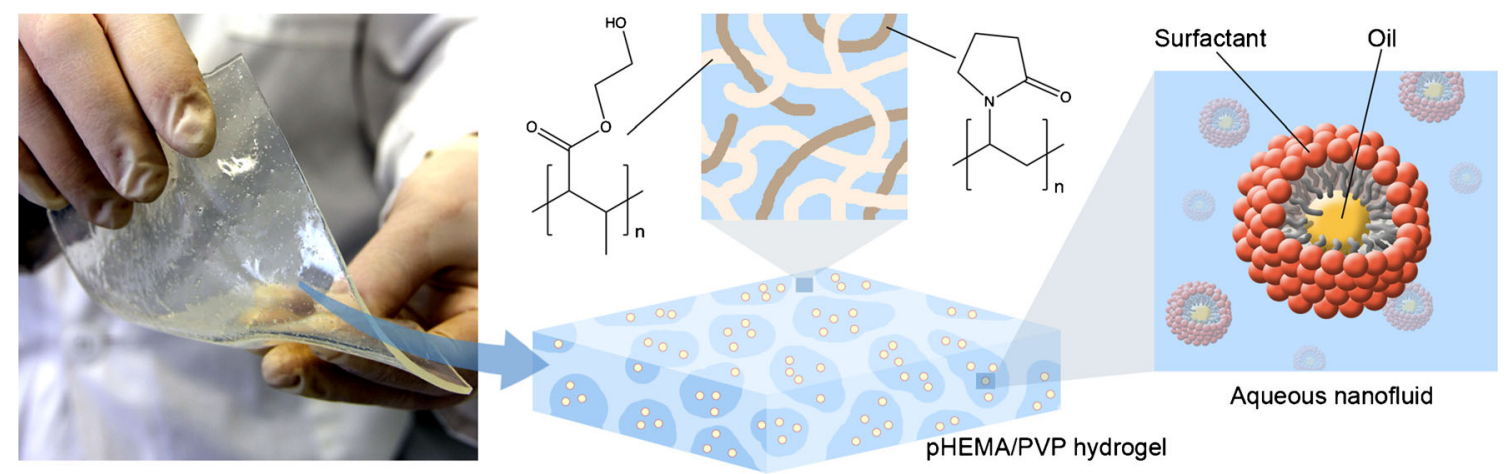

pHEMA/PVP hydrogel

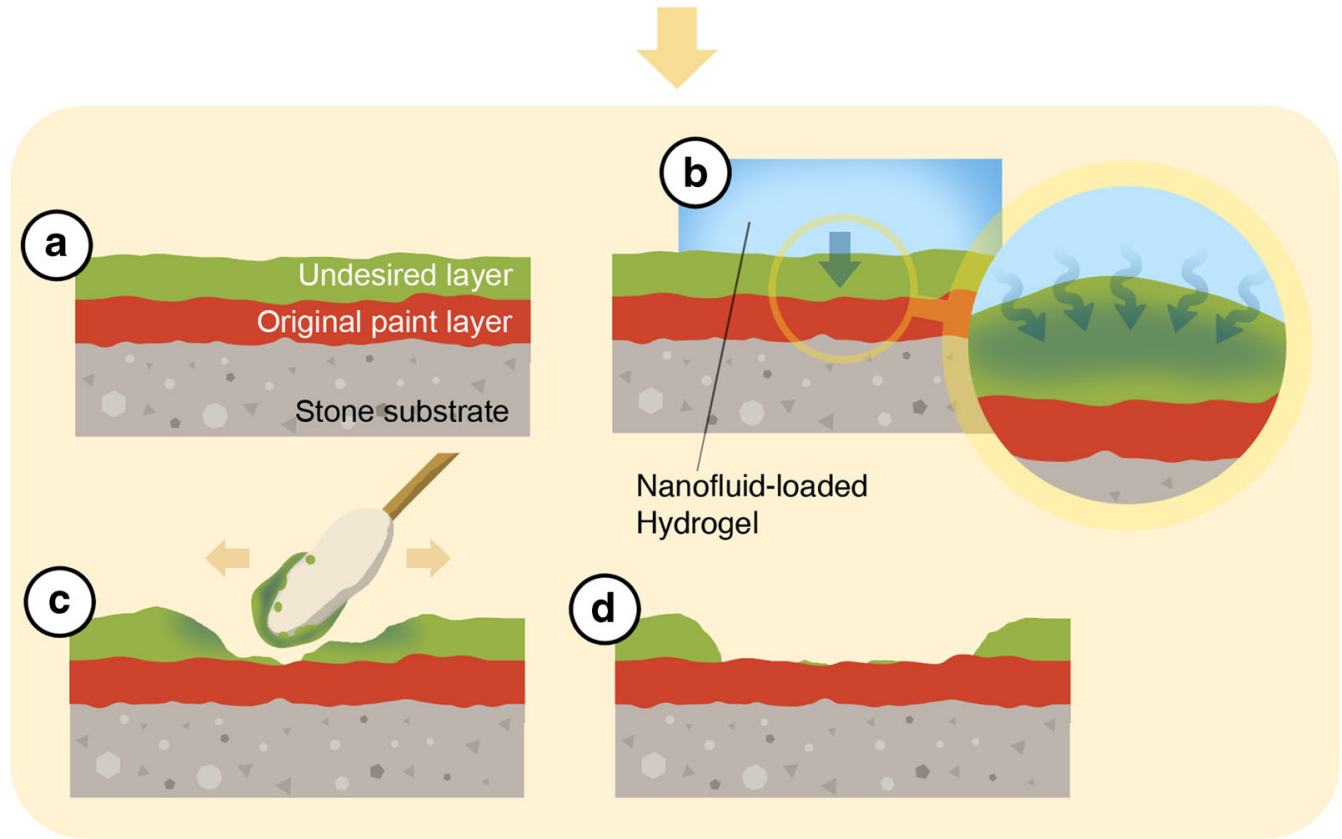

Fig. 2 Top, the cartoon represents schematically the porous structure of a pHEMA/PVP hydrogel (which is shown in the photograph, handled in the form of a 2-mm-thick sheet). The polymeric network of the gel is composed of a semi-interpenetrated mesh composed of poly(hydroxyethyl methacrylate) and poly(vinyl pyrrolidone). Finally, when the gel is loaded with a nanofluid, micelles diffuse through the porosity of the gel itself. A generic nanofluid is represented as composed of surfactant/oil supramolecular aggregates dispersed in water. Bottom, the box shows the process observed for selective removal of an undesired paint layer using, as proposed, a nanofluid-loaded highly retentive chemical hydrogel. $a$ The green paint layer was laid on top of the original red paint layer, which is the one that needs to be preserved. $b$ A nanofluid-loaded hydrogel is put in contact with the green paint layer for the selected application time. The top paint layer swells as a consequence of solvent migration from the nanofluid/gel cleaning system. By varying application time and retentive properties of the gel, it is possible to tune the depth of penetration of solvents, trying not to damage the red original layer. $c \mathrm{~A}$ gentle mechanical action is performed with a humid cotton swab on the swollen polymer. Removal is now easier and controlled. $d$ The red original paint layer is again visible. Repeating the same procedure, it is possible to clean the whole surface 
propylene carbonate, or the selected nanofluid. It was shown that with none of these free liquids, it was possible to obtain a controlled action, i.e., migration of solvents into the paint layers was too fast and the blue paint layer was swollen almost at the same time as the white top paint layer.

Then, several applications of the nanofluid-loaded gel were repeated, in order to find the most suitable combination of application times, i.e., long enough to swell the paint layer to be removed, while not affecting the layer to be preserved. In Fig. 1, two different test areas were selected and evidenced to be treated as two reference results. Top-right area represents the first of the cleaning tests and shows the presence of several residues of white paint (see the relative micrograph). Here, the removal was uneven and it is likely that most likely the nanofluid-loaded gel was not left in contact with the white paint long enough to produce a homogeneous swelling.

In the highlighted left treated area (see Fig. 1), conversely, it can be noted that satisfactory results are obtained only by adjusting application times and refining the mechanical removal of the swollen paint. FT-IR analyses and micrograph report confirm that the top paint layer was completely removed, while the original paint layer was not significantly damaged.

In particular, looking at the FT-IR data reported in Fig. 1, it can be noticed that the microreflectance spectra of the cleaned area and the blue paint are almost identical, while the spectrum of the white paint is significantly different with respect to the other two. This means that residues of white paint are either absent or below the minimum detection limit of the analytical technique.

The tests performed show that selective removal is achievable, even when chemical nature of the two paints is almost identical, and that nanofluid-loaded hydrogels actually make the cleaning procedure more controlled, operating a "layer-by-layer" gradual removal. In Fig. 2 a cartoon describes the selective removal process through which the gel-nanofluid combined system works.

\section{Conclusions}

Removal of overpaintings and graffiti from street art is a relatively new topic in the field of conservation of cultural heritage; therefore, at present, few case studies and almost no publications can be found on this issue. However, in view of the growing interest on street art in the recent years, it can be predicted that the need to intervene with selective cleaning operations on paintings realized with unconventional modern/ contemporary materials will substantially increase in the future.

Chemical removal cannot rely on chemical selectivity, because, unfortunately, in most cases, the binder of the undesired paint layer has the same nature of the original one to be preserved. Therefore, the most promising solution, at present, is the combination of smart nanostructured liquid systems confined in a solid scaffold ("soft," working with gels) that allows limiting the action of the fluid at the contact surface between the artwork and the cleaning system itself.

The use of a pHEMA/PVP hydrogel loaded with a nanofluid based on propylene carbonate and ethyl acetate for the selective removal of a layer of acrylic white paint from an acrylic blue paint laid over a mortar sample gave satisfactory results. It was shown that selective removal is achievable combining the suitable application times of the nanofluid-loaded hydrogel with a gentle mechanical action, by means of which the swollen paint is removed from the treated surface. The original paint layer was left unaltered, and no residues of white paint were found on the cleaned area.

In conclusion, we proposed a new methodology to approach a growing conservative issue, such as selective removal of graffiti and overpaintings from street art, which exploits the combination of nanofluids and highly retentive chemical hydrogels. The combination of these products of colloids science made possible to overcome the limitations of traditional cleaning techniques in this context.

Acknowledgements Margherita Alterini is acknowledged for the assistance with laboratory tests on removal of overpaintings from modern paints laid on mortar samples. Yvonne Shashoua and Isabelle Brajer are kindly acknowledged for fruitful discussion about this topic and for providing us with useful information about this new conservative issue. CSGI is acknowledged for partly funding this work, which was otherwise supported by the European Union (CORDIS)-Project NANORESTART (H2020-NMP-21-2014/646063).

\section{Compliance with ethical standards}

Conflict of interest The authors declare that they have no conflict of interests.

\section{References}

1. Sanmartín P, Cappitelli F, Mitchell R. Current methods of graffiti removal: a review. Constr Build Mater. 2014;71:363-74. doi:10.1016/j.conbuildmat.2014.08.093.

2. Whitford MJ (1992) Getting rid of graffiti: a practical guide to graffiti removal and anti-graffiti protection. Taylor \& Francis

3. Pozo-Antonio JS, Ramil A, Fiorucci MP, López AJ, Rivas T. The use of hyperspectral imaging technique to detect the most suitable graffiti-cleaning procedure. Color Res Appl. 2016;41:308-12. doi:10.1002/col.22032.

4. Pozo-Antonio JS, Rivas T, López AJ, Fiorucci MP, Ramil A. Effectiveness of granite cleaning procedures in cultural heritage: a review. Sci Total Environ. 2016;571:1017-28. doi:10.1016/j. scitotenv.2016.07.090.

5. Pozo-Antonio JS, Rivas T, Fiorucci MP, López AJ, Ramil A. Effectiveness and harmfulness evaluation of graffiti cleaning by mechanical, chemical and laser procedures on granite. Microchem J. 2016;125:1-9. doi:10.1016/j.microc.2015.10.040.

6. Chapman S. Laser technology for graffiti removal. J Cult Herit. 2000;1(Supplement 1):S75-8. doi:10.1016/S12962074(00)00153-9. 
7. Carvalhão M, Dionísio A. Evaluation of mechanical soft-abrasive blasting and chemical cleaning methods on alkyd-paint graffiti made on calcareous stones. J Cult Herit. 2015;16:579-90. doi:10.1016/j.culher.2014.10.004.

8. Siano S, Osticioli I, Pavia A, Ciofini D. Overpaint removal from easel paintings using an LQS Nd:YAG laser: the first validation study. Stud Conserv. 2015;60:S49-57. doi:10.1179/0039363015Z. 000000000207.

9. Apostol I, Damian V, Garoi F, Iordache I, Bojan M, Apostol D, Armaselu A, Morais PJ, Postolache D, Darida I. Controlled removal of overpainting and painting layers under the action of UV laser radiation. Opt Spectrosc. 2011;111:287. doi:10.1134/ S0030400X11080054.

10. Ciofini D, Osticioli I, Pavia A, Siano S. Removal of overpaintings from easel paintings using LQS Nd:YAG laser. Appl Phys A Mater Sci Process. 2014;117:341-6. doi:10.1007/s00339-014-8318-2.

11. Baglioni P, Berti D, Bonini M, Carretti E, Dei L, Fratini E, Giorgi R. Micelle, microemulsions, and gels for the conservation of cultural heritage. Adv Colloid Interf Sci. 2014;205:361-71. doi:10.1016/j. cis.2013.09.008

12. Pizzorusso G, Fratini E, Eiblmeier J, Giorgi R, Chelazzi D, Chevalier A, Baglioni P. Physicochemical characterization of acrylamide/bisacrylamide hydrogels and their application for the conservation of easel paintings. Langmuir. 2012;28:3952-61. doi:10.1021/la2044619.

13. Baglioni M, Bartoletti A, Bozec L, Chelazzi D, Giorgi R, Odlyha M, Pianorsi D, Poggi G, Baglioni P. Nanomaterials for the cleaning and $\mathrm{pH}$ adjustment of vegetable-tanned leather. Appl Phys A Mater Sci Process. 2016;122:114. doi:10.1007/s00339-015-9553-x.

14. Baglioni P, Chelazzi D. Nanoscience for the conservation of works of art: Royal Society of Chemistry; 2013.

15. Bonelli N, Chelazzi D, Baglioni M, Giorgi R, Baglioni P. Confined aqueous media for the cleaning of cultural heritage: innovative gels and amphiphile-based nanofluids. In: Dillmann P, Bellot-Gurlet L, Nenner I, editors. Nanosci. Cult: Herit. Atlantis Press; 2016. p. 283-311.

16. Domingues JAL, Bonelli N, Giorgi R, Fratini E, Gorel F, Baglioni P. Innovative hydrogels based on semi-interpenetrating $\mathrm{p}(\mathrm{HEMA}) / \mathrm{PVP}$ networks for the cleaning of water-sensitive cultural heritage artifacts. Langmuir. 2013;29:2746-55. doi:10.1021/la3048664.

17. Giorgi R, Baglioni M, Berti D, Baglioni P. New methodologies for the conservation of cultural heritage: micellar solutions, microemulsions, and hydroxide nanoparticles. Acc Chem Res. 2010;43:695-704. doi:10.1021/ar900193h.

18. Baglioni M, Rengstl D, Berti D, Bonini M, Giorgi R, Baglioni P. Removal of acrylic coatings from works of art by means of nanofluids: understanding the mechanism at the nanoscale. Nano. 2010;2:1723. doi:10.1039/c0nr00255k.

19. Baglioni M, Berti D, Teixeira J, Giorgi R, Baglioni P. Nanostructured surfactant-based systems for the removal of polymers from wall paintings: a small-angle neutron scattering study. Langmuir. 2012;28:15193-202. doi:10.1021/la303463m.

20. Domingues J, Bonelli N, Giorgi R, Baglioni P. Chemical semi-IPN hydrogels for the removal of adhesives from canvas paintings. Appl Phys A Mater Sci Process. 2013;114:705-10. doi:10.1007/s00339013-8150-0

21. Domingues J, Bonelli N, Giorgi R, Fratini E, Baglioni P. Innovative method for the cleaning of watersensitive artifacts: synthesis and application of highly retentive chemical hydrogels. Int J Conserv Sci. 2013;4:715-22.

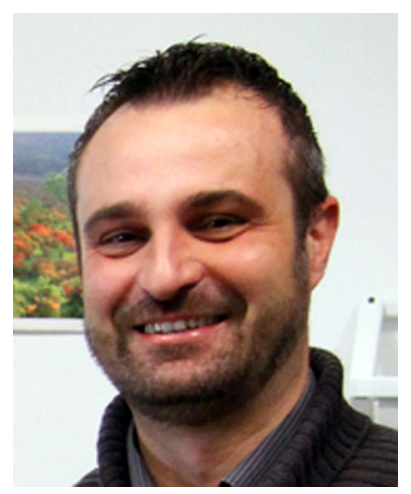

Rodorico Giorgi, $\mathrm{PhD}$ in Science for Cultural Heritage Conservation at the University of Florence and $\mathrm{BS}$ in Chemistry, is currently an associate professor of Chemistry at the University of Florence and CSGI. Giorgi's background is in colloids science. His main research interests are the development of methodologies for the conservation of cultural heritage materials such as wall and easel paintings, stone, paper, and archeological wood. Giorgi is the author of about 100 publications in the field of science for conservation.

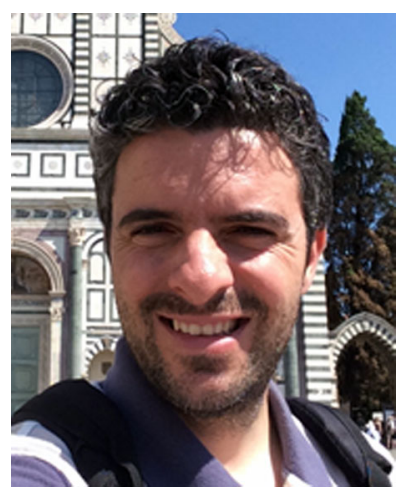

Michele Baglioni got his $\mathrm{PhD}$ in Science for Conservation of Cultural Heritage in 2010 . He is currently a post-doctoral research fellow at the CSGI and Chemistry Department, University of Florence. His interests and expertise are related to the development and characterization of soft-matter systems for the cleaning of works of art, such as nanostructured fluids (i.e., microemulsions and micelles) and gels.

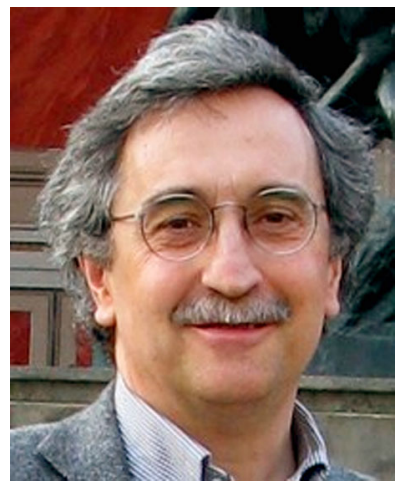

Piero Baglioni has been the Chair of Physical Chemistry in the Department of Chemistry at the University of Florence since 1994 and is an MIT affiliate. He was appointed as visiting scientist/ professor by the Department of Chemistry of the University of Houston, the Weizmann Institute, the Collège de France, and MIT. He is the Director of the National Center for Colloids and Nanosciences (CSGI), and he is on the advisory boards of several international journals and a member of the scientific board of several national and international institutions and societies. He is the author of more than 350 publications in books and largely diffused international journals. He is also the author of 21 patents. In the field of conservation, he is a pioneer in the application of colloids and soft matter to the conservation of cultural heritage. He has produced several innovative methods for the consolidation and cleaning of paintings and the deacidification of historical documents. 